ISSN 2306-1561

Automation and Control in Technical Systems (ACTS)

2014, No 1.2(9), pp. 158-164.

DOI: $10.12731 / 2306-1561-2014-1-29$

\title{
Control model components connected dosing construction mixtures
}

\section{Marsov Vadim Israilevich}

Russian Federation, Doctor of Technical Sciences, Professor, Department of «Automation Industrial Process».

State Technical University - MADI, 125319, Russian Federation, Moscow, Leningradsky prospekt, 64. Tel.: +7 (499) 151-64-12. http://www.madi.ru

\section{Kolbasin Alexander Markovich}

Russian Federation, Ph.D., Associate Professor, Department of «Automation Industrial Process».

State Technical University - MADI, 125319, Russian Federation, Moscow, Leningradsky prospekt, 64. Tel.: +7 (499) 151-64-12. http://www.madi.ru

\section{alex123456789.a@yandex.ru}

\section{Abdulkhanova Marina Yurievna}

Russian Federation, Ph.D., Associate Professor, Department of «Automation Industrial Process».

State Technical University - MADI, 125319, Russian Federation, Moscow, Leningradsky prospekt, 64. Tel.: +7 (499) 151-64-12. http://www.madi.ru

\section{Dinh An Ninh}

People's Republic of China, Postgraduate Student, Department of «Automated Control Systems».

State Technical University - MADI, 125319, Russian Federation, Moscow, Leningradsky prospekt, 64. Tel.: +7 (499) 151-64-12. http://www.madi.ru

Abstract. In article developed a coherent model of continuous dosing and an algorithm for compensation of dosing errors, reflecting a fundamentally new approach to reducing errors dosing construction mixtures.

Keywords: dosing, control, processes and manufacturing industries, extreme control system (ECS), construction mixtures. 
ISSN 2306-1561

Автоматизация и управление в технических системах (АУТС) 2014. - №1.2(9). - C. 158-164.

DOI: $10.12731 / 2306-1561-2014-1-29$

\section{УДК 681.3}

\section{Модель управления связным дозированием компонентов строительных смесей}

\section{Марсов Вадим Израилевич}

Российская Федерация, доктор технических наук, профессор кафедры «Автоматизация производственных процессов».

ФГБОУ ВПО «Московский автомобильно-дорожный государственный технический университет (МАДИ)», 125319, Российская Федерация, г. Москва, Ленинградский проспект, д.64, Тел.: +7 (499) 151-64-12, http://www.madi.ru

\section{Колбасин Александр Маркович}

Российская Федерация, кандидат технических наук, доцент кафедры «Автоматизация производственных процессов».

ФГБОУ ВПО «Московский автомобильно-дорожный государственный технический университет (МАДИ)», 125319, Российская Федерация, г. Москва, Ленинградский проспект, д.64, Тел.: +7 (499) 151-64-12, http://www.madi.ru

alex123456789.a@yandex.ru

\section{Абдулханова Марина Юрьевна}

Российская Федерация, кандидат технических наук, доцент кафедры «Автоматизация производственных процессов».

ФГБОУ ВПО «Московский автомобильно-дорожный государственный технический университет (МАДИ)», 125319, Российская Федерация, г. Москва, Ленинградский проспект, д.64, Тел.: +7 (499) 151-64-12, http://www.madi.ru

\section{Динь Ан Нинь}

Китайская Народная Республика, аспирант кафедры «Автоматизация производственных процессов».

ФГБОУ ВПО «Московский автомобильно-дорожный государственный технический университет (МАДИ)», 125319, Российская Федерация, г. Москва, Ленинградский проспект, д.64, Тел.: +7 (499) 151-64-12, http://www.madi.ru

Аннотация. В статье разработана модель связного непрерывного дозирования и предложен алгоритм компенсации ошибок дозирования, отражающие принципиально новый подход к уменьшению погрешностей дозирования компонентов строительных смесей. 
Ключевые слова: дозирование, управление, технологические процессы и производства, промышленные предприятия, системы экстремального регулирования (СЭР), строительные смеси.

\section{1. Введение}

Чтобы удовлетворить более высоким технологическим требованиям, предъявлемым к дозаторам непрерывного действия и обеспечить тем самым наибольшую эффективность процессов смесеобразования, необходимо предложить способы автоматизации, придающие системам дозирования принципиально новые качественные свойства [1 - 6]. Это, в первую очередь, относится к снижению погрешностей дозирования компонентов за счет изменения принципов управления процессами дозирования с переходом от индивидуального управления отдельными дозаторами к связному управлению взаимозависимой по ошибкам дозирования отдельных доз материала системой дозирования [7 - 11].

\section{2. Поддержание качества смеси и изменение ошибок дозирования}

Изменение дисперсий $D\left\{\delta_{j}\right\}$ ошибок дозирования $\delta_{j}$ и математических ожиданий распределения показателей качества $Q_{i}$ отдельных компонентов взаимосвязано. Наличие такой взаимосвязи через глобальный критерий и корреляционные функции $K\left\{y_{i j}, y_{i l}\right\}$ статистических характеристик вкладов $i$-ых показателей качества $j$ - ого и $l$ - ого компонентов $y_{i j}, y_{i l}$ в отдельные показатели качества смеси, дает эффективный механизм реализации процесса многокомпонентного дозирования - связного дозирования. Это новейшее направление в управлении циклическими и непрерывными процессами приготовления многокомпонентных смесей является эффективным способом изменения их технико-экономических показателей, снижения стоимости и повышения качества готового продукта.

Задача управления качеством строительных смесей может быть сформулирована как задача максимизации вероятности попадания их качественных показателей $Q_{i}$ в заданную технологическими нормами область, описанную верхней $Q_{i}{ }^{B}$ и нижней $Q_{i}{ }^{H}$ границами изменения $Q_{i}$ :

$$
\max P\left[Q_{i}^{H}+\aleph_{i} \sqrt{D\left\{Q_{i}\left(\Delta x_{j}\right)\right\}} \leq Q_{i}\left(x_{j}\right) \leq Q_{i}^{B}-\aleph_{i} \sqrt{D\left\{Q_{i}\left(\Delta x_{j}\right)\right\}}\right]
$$

По окончании циклического дозирования $k$ компонентов часть слагаемых в выражении $Q_{i}=\sum_{j=1}^{n} a_{i j} x_{j}$, где $a_{i j}$ - коэффициенты влияния $i$-го свойства $j$-го компонента массой $x_{j}$ на качество смеси, принимает не предполагаемые, а реальные значения. Тогда: 


$$
Q_{i}=\sum_{j=1}^{n} a_{i j}\left(x_{j}^{0}+\Delta x_{j}\right)+\sum_{j=k+1}^{n} a_{j} x_{j}
$$

где $x_{j}^{0}, \Delta x_{j}$ - заданная масса и ошибка дозирования ј-го компонента, задача оптимизации приобретает вид:

$$
\begin{aligned}
& \min \left[I\left(x_{j}\right) / Q_{i}^{H}+\aleph_{i} \sqrt{\left.D\left\{\sum_{j=1}^{n} a_{j} \Delta x_{j}\right)\right\}}+\sum_{j=1}^{k} a_{i j} x_{j} \leq \sum_{j=k+1}^{n} a_{i j} x_{j} \leq Q_{i}^{B}--\aleph_{i} \sqrt{\left.D\left\{\sum_{j=1}^{n} a_{j} \Delta x_{j}\right)\right\}}\right. \\
& -\sum_{j=1}^{k} a_{i j} x_{j} \\
& \quad ; \\
& \left.\Delta x_{j}+x_{j}^{0}=\bar{x}_{j}(j=\overline{1}, \bar{k}) ; \sum_{j=1}^{n} \gamma_{j}=1 ; \gamma_{j} \geq 0\right] \\
& \text { где }-\sum_{j=1}^{k} a_{i j} x_{j}-\text { изменение качественных показателей смеси за счет учета }
\end{aligned}
$$

отдозированных компонентов; $\gamma_{i}$ - долевые соотношения компонентов смеси; $\boldsymbol{\aleph}_{i}$ постоянный коэффициент.

Поддержание качества смеси на расчетном уровне достигается коррекцией отклонений вкладов в параметры качества отдозированных компонентов, изменением вкладов последующих. Для этого корректируются уставки дозаторов не отдозированных компонентов в зависимости от ошибок дозирования предыдущих.

Введение в модель детерминированного аналога вероятностных ограничений $\aleph \sqrt{D\{Q\}}$, сужая область допустимого существования переменных, позволяет, находить наилучшие сочетания значений $x_{j}$ в более узкой области их изменения. Возможная, хотя и с малой вероятностью, некоторая потеря качества при выходе ошибок дозирования за пределы оптимизации, окупается повышением качества смеси в остальных циклах управления, где эти отклонения отсутствуют.

\section{3. Модель связного управления многокомпонентным дозированием}

Использование дозаторов непрерывного действия при производстве строительных смесей, связано с интегрированием ошибок дозирования $\int_{0}^{T} \Delta \Theta_{j} d t$ (где $\Delta \Theta$ - отклонение производительности дозатора от номинала) за принятый условный цикл управления $T$, составляющий часть полного времени цикла набора дозы выбранного компонента, позволяет скорректировать граничные значения качества $Q^{H}, Q^{B}$ и оптимизировать состав смеси для следующего условного цикла дозирования уже в новых границах:

$$
Q_{i}^{H}+\aleph_{i} \sqrt{D\left\{Q_{i}\right\}}+\sum_{j=1}^{n} a_{j} \int_{0}^{T} \Delta \Theta_{j} d t \leq Q_{i} \leq Q_{i}^{B}-\aleph_{i} \sqrt{D\left\{Q_{i}\right\}}-\sum_{j=1}^{n} a_{j} \int_{0}^{T} \Delta \Theta_{j} d t .
$$


Для дозаторов непрерывного действия, ошибки дозирования, интегрируясь в процессе измерений за интервал условного цикла $T$, определяют сдвиг кривой распределения случайных значений $Q_{i}$ относительно центра рассеивания на величину его математического ожидания $M\left\{\sum_{j=1}^{n} a_{i j} \Delta x_{j}\right\}$ в границах:

$$
Q_{i}{ }^{H}+\aleph_{i} \sqrt{D\left\{Q_{i}\left(\Delta x_{j}\right)\right\}} \leq Q_{i}\left(x_{j}\right)+M\left\{Q_{i}\left(\Delta x_{j}\right)\right\} \leq Q_{i}^{B}-\aleph_{i} \sqrt{D\left\{Q_{i}\left(\Delta x_{j}\right)\right\}} .
$$

Таким образом, модель связного управления многокомпонентным дозированием для n-ого интервала измерений, в отличие от моделей $(3,5)$, будет иметь вид:

$$
\begin{aligned}
& \min \left[F(\bar{x}) / Q_{i}^{H}+\aleph_{i} \sqrt{D\left\{Q_{i}\right\}} \leq Q_{i}+M\left\{\sum_{j=1}^{n} a_{i j} \Delta x_{j}\right\} \leq Q_{i}^{B}-\aleph_{i} \sqrt{D\left\{Q_{i}\right\}}\right] \\
& D\left\{Q_{i}\left(Y_{i}, \vec{\delta}\right)\right\}=\sum_{j=1}^{n}\left[D\left\{y_{i j}\right\}+2 \sum_{l=1}^{n} K\left\{y_{i j}, y_{i l}\right\}+10^{-4}\left(D\left\{y_{i j}\right\}+M^{2}\left\{y_{i j}\right\} D\left\{\delta_{j}\right\}\right]\right. \\
& Q=\sum_{j=1}^{n} a_{i} x_{j}, \sum \gamma_{j}=1 \\
& \text { где } M\left\{\sum_{j=1}^{n} a_{i j} \Delta x_{j}\right\}-\text { математическое ожидание отклонения массы материала за }
\end{aligned}
$$

интервал измерений; $\gamma_{j}$ - относительное содержание $j$-го компонента в смеси,

$K\left\{y_{i j}, y_{i l}\right\}$ - коэффициент корреляции.

Дисперсия параметров $Q_{i}$ является функцией статистических характеристик вкладов $y_{i j}$ и случайных ошибок дозирования. Дисперсия $D\left\{\delta_{i j}\right\}$ определяется погрешностью всей системы измерения расхода.

Вероятность $P\left\{\vec{Q}\left(Y_{i}, \vec{\delta}\right)\right\}$ попадания качества смеси в область ограничений $\Omega$, является монотонно убывающей функцией дисперсий параметров качества $Q_{i}$ с максимумом, равным $P^{0}$. Максимуму значения вероятности соответствует минимальное значение дисперсий $Q_{i}$, получаемое при $\delta_{j}=0$ :

$$
D\left\{Q_{i}\left(Y_{i}\right)\right\}=\sum_{j=1}^{n}\left[D\left\{y_{i j}\right\}+2 \sum_{l=1}^{n} K\left\{y_{i j}, y_{i l}\right\}\right] .
$$

Ошибки дозирования каждого $j$-го компонента при независимом способе дозирования, когда коэффициент корреляции $K\left\{y_{i j}, y_{i l}\right\}=0$, уменьшает вероятность $P^{0}$ на величину:

$$
\Delta P_{j}=P_{-}^{0} P\left\{\vec{Q}\left(Y_{i}, \delta_{j}\right) \in \Omega,\right\},
$$


где $P\left\{\vec{Q}\left(Y_{i}, \delta_{j}\right) \in \Omega,\right\}$ - вероятность попадания качественных показателей смеси в область $\Omega$, описываемую через интеграл Лапласа; $D\left\{Q_{i}\left(Y_{i}, \delta_{j}\right)\right\}$ - дисперсия, рассчитываемая по формуле по формуле:

$$
D\left\{Q_{i}\left(Y_{i}, \delta_{j}\right)\right\}_{=} D\left\{Q_{i}\left(Y_{i}\right)\right\}_{+} 10^{-4}\left(D\left\{y_{i j}\right\}+M^{2}\left\{y_{i j}\right\}\right) D\left\{\delta_{j}\right\} .
$$

Общее снижение вероятности $\Delta P^{n}$ ошибки независимого дозирования $\mathrm{n}$ компонентов равно: $\Delta P^{n}=\sum_{j=1}^{n} \Delta P_{j}$.

При связном дозировании потенциальные возможности системы снижения технологических ошибок дозирования расширяются за счет варьирования величин взаимокорреляционных моментов в ограничениях модели (6).

Значение $\Delta P_{j}$ может быть уменьшено до $\Delta P_{j l}$, изменением дисперсий параметров $Q_{i}$.

Возможный диапазон изменения дисперсий $Q_{i}$ определяется неравенством:

$$
D\left\{Q_{i}\left(Y_{i}\right)\right\}_{\leq} D\left\{Q_{i}\left(Y_{i}, \delta_{j}\right)\right\}_{\leq} D\left\{Q_{i}\left(Y_{i}, \vec{\delta}_{j}\right)\right\}
$$

где $D\left\{Q_{i}\left(Y_{i}, \delta_{j}\right)\right\}$ - дисперсия параметров качества смеси минимальная при отсутствии ошибок дозирования и максимальная при независимом способе дозировании компонентов.

Представление непрерывного процесса дозирования последовательностью из $\mathrm{n}$ интервалов протяженностью $T$, позволяет решить задачу повышения точности измерений расхода, учитывая свойства измерительной системы при входных стандартных (скачок, импульс) и случайных воздействиях.

Серия импульсных или скачкообразных воздействий за время условного периода $T$ дает для дозаторов величину, близкую к математическому ожиданию отклонения измеренного значения массы материала на ленте от задания. Если параметры $Q_{i}^{0}$ независимы и распределены по нормальному закону, а кривые их плотности распределения симметричны относительно математического ожидания, то появление ошибок дозирования смещает кривые плотности распределения и уменьшает вероятность попадания качества массы в область $\Omega$. Поэтому в конце каждого условнопостоянного интервала непрерывного процесса дозирования необходимо откорректировать измеренные значения массы материала на величины их мгновенного отклонения $M\{\delta\}$ от среднего по времени нахождения на ленте транспортера в момент измерений.

При связном дозировании появляется возможность использовать информацию о текущих значениях измеренных масс отдельных компонентов для достижения максимального значения глобального критерия всей системы многокомпонентного дозирования в соответствии с моделью (4), варьируя величины $D\left\{Q_{i}\left(Y_{i}, \delta_{j}\right)\right\}$ в области 
ограничений неравенства (5) в конце каждого условно-постоянного интервала измерений $T$.

\section{4. Заключение}

Разработанная модель связного непрерывного дозирования отражает принципиально новый подход к уменьшению погрешностей дозирования в процессе набора заданных доз материала за счет связного способа дозирования.

\section{Список информационных источников}

[1] Марсов В.И., Тихонов А.Ф., Бокарев Е.И. Дозирующие системы непрерывнлого действия нового поколения // Механизация строительства. - 2012. - №3. - С. 2730 .

[2] Васильев Ю.Э., Марсов В.И., Гиляровский Т.В. Оптимизация динамических процессов комбинированной системы дозирования // Механизация строительства. - 2012. - №8. - С. 40-43.

[3] Марсов В.И., Суэтина Т.А., Колбасин А.М., Тан Цзиюя. Алгоритм управления оптимальной системы пневмодозирования сыпучих материалов // Автоматизация и управление в технических системах. - 2013. - № 1(3). - С. 39-44.

[4] Колбасин А.М., Тан Цзиюя, Марсова Е.В., Шухин В.В. Система экстремального регулирования потока материала в пневмосистеме // Автоматизация и управление в технических системах. - 2013. - № 1(3). - С. 44-48.

[5] Марсов В.И., Колбасин А.М., Тан Цзиюя, Шухин В.В. Система оптимального регулирования процессом пневмотранспортирования // Автоматизация и управление в технических системах. - 2013. - № 1(3). - С. 48-52.

[6] Марсов В.И., Колбасин А.М., Сарычев И.Ю., Курилин А.В. Методы улучшения качественных характеристик системы экстремального регулирования // Автоматизация и управление в технических системах. - 2013. - № 3(5). - С. 3-6.

[7] Остроух А.В. Автоматизация и моделирование работы предприятий по строительству промышленных объектов: дис. ... д-ра техн. наук: 05.13.06: защищена 07.04.09: утв. 19.06.09. - М., 2009. - 357 с.

[8] Остроух А.В. Автоматизация и моделирование работы предприятий по строительству промышленных объектов: автореф. дис. ... д-ра техн. наук: 05.13.06. - М., 2009. - 43 с.

[9] Остроух А.В. Исследование начального периода моделирования на точность среднеинтегральной оценки имитационных моделей / А.В. Остроух, А.А. Солнцев, Н.В. Солдатов, К.А. Новицкий, П.С. Якунин // Вестник МАДИ. - 2010. Вып. 2(21). - С. 61-65.

[10] Остроух А.В., Тянь Юань. Современные методы и подходы к построению систем управления производственно-технологической деятельностью промышленных предприятий // Автоматизация и управление в технических системах. - 2013. - № 1(3). - C. 29-31.

[11] Остроух А.В. Интеграция компонентов системы мониторинга / А.В. Остроух, Юань Тянь // Молодой ученый. - Чита: ООО «Издательство Молодой ученый», 2013. - №10. - С. 182-185. 